\title{
4th time is a charm: Peritoneal tuberculosis and how difficult is to reach it
}

\author{
Ana Gonçalves, Helena Maurício, Cristiana Batouxas, Miriam Blanco \\ Serviço de Medicina, Hospital de Bragança. Portugal
}

\begin{abstract}
Tuberculosis has an increased incidence in the last few years due to the phenomenon of migration and remains a public health problem in certain regions of the world. The abdominal form is the sixth leading cause of extrapulmonary TB, after lymphatic, genitourinary, osteoarticular, miliary and meningeal. Usually, is more prevalent in <40years old females and due to its nonspecific clinical manifestations remains a clinical challenge for most physicians. We present the case of 42--year--old female with peritoneal tuberculosis (PTB). The patient suffered from abdominal pain and increasing abdominal perimeter 13 days prior to admission. She went to the emergency department four times in 10 days and was discharged with multiple diagnosis. The diagnosis was established on the basis of clinical features, findings from an abdominal tomography scan and histopathological analysis of peritoneal biopsy. The aim of this review is to expose the struggle experience diagnosing such a challenging disease.
\end{abstract}

Key words: Peritoneal Tuberculosis, Tuberculosis, Abdominal pain, Ascitis, Exploratory Laparotomy.

\section{Introduction}

Peritoneal tuberculosis is one of the forms of abdominal tuberculosis, and can be located in any site of the gastrointestinal and genitourinary tract, solid organs, abdominal aorta and linf nodes. About $30 \%$ of cases of extrapulmonary tuberculosis and $20 \%$ of all causes of ascites occur in developing countries. This form of tuberculosis is rarely associated with the active form of pulmonary disease, sometimes presenting with nonspecific symptoms, and for this reason has been described by some authors as "the great mimic". Peritoneal involvement is often accompanied by abdominal pain (80--95\%) often associated with fever(40--90\%), weight loss (40-90\%)and anorexia (30\%), and this combination may occur for many months. Among the known risk factors are patients diagnosed with cirrhosis, human immunodeficiency virus infection, diabetes mellitus, malignancies, immunosuppressive patients, and patients undergoing dialysis. In most cases, the infection occurs through the reactivation of a

Fig. 1. Chest $X$ Ray revealed no infiltrations

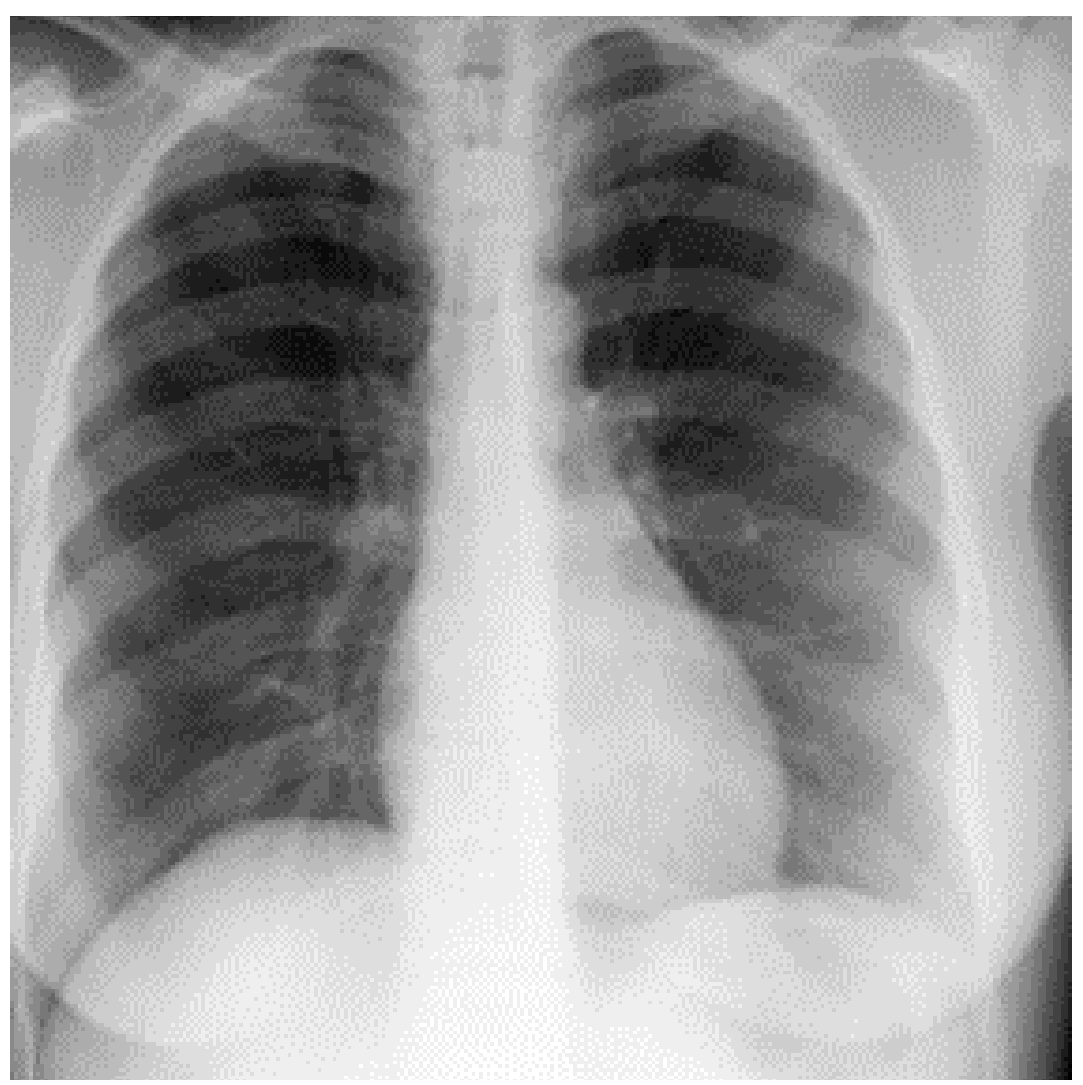


latent focus located in the peritoneum (through hematogenous dissemination of a pulmonary focus), hematogenous through an active pulmonary focus or miliary tuberculosis, with less invasion of the cavity peritoneal disease through intestinal infections or genitourinary tuberculosis. Peritoneal TB has three types: the wet type with ascites, the encysted type with abdominal swelling and the fibrotic type with abdominal masses.

\section{Case Report}

A 42--year--old female, with no history of disease, was admitted to the emergency department with a 13days history of abdominal pain and low fever. After four episodes of medical consultation, the patient was admitted for etiological study. She was feverish, with complaints of malaise and anorexia with weight loss of $7 \mathrm{Kg}$. The patient's abdomen was blown, soft with normal bowel sounds and ascites. The initial laboratory studies showed low hemoglobin $(10,7 \mathrm{~g} / \mathrm{dL})$, normal white blood cells $(4,987 / \mu l)$ increased erythrocyte sedimentation rate $(51 \mathrm{~mm} / \mathrm{sec})$, protein C reaction (39mg/dL) and CA--125 (346 IU/mL). Hepatic and renal function were normal. HIV and hepatotrophic viruses were negative. A tuberculin skin test was negative.

Chest X--Ray did not present any infiltrations (fig 1). A pelvic--abdominal computed tomography (fig 2 and 3 ) showed ascites of moderate volume and a pelvic left adnexial tubular lesion with serpiginous morphology with hypothesis of peritoneal tuberculosis with tubular involvement. A diagnostic paracentesis was performed and revealed exsudate findings. The patient underwent exploratory laparoscopic with peritoneal biopsy which confirmed peritoneal tuberculosis. The patient received fourfold antituberculous treatment with isonizid, rifampicin, pyrazinamide and ethambutol and was discharged from the hospital to continue treatment at home for 6 months. A regular follow--up was performed.

\section{Bibliography}

1. Flores-Alvarez E, Tello-Brand SE, Lopez-Lopez F, Rivera-Barragan V. Peritoneal tuberculosis. Report of seven cases. Cir Cir. 2010;78:67-71.

2. Sanai FM, Bzeizi KI. Systematic review: Tuberculous peritonitis - presentingfeatures, diagnostic strategies and treatment. Aliment Pharmacol Ther. 2005;22:685-700.

3. Malik A, Saxena NC. Ultrasound in abdominal tuberculosis. Abdom Imaging 2003:28:574-579.

4. Sharma MP, Bhatia V. Abdominal tuberculosis. Indian J Med Res. 2004;120:305315

5. Marshall JB. Tuberculosis of the gastrointestinal tract and peritoneum. Am J Gastroenterol. 1993:88:989-999.

6. Diagnostic Standards and Classification of Tuberculosis in Adults and Children. This oficial statement of the American Thoracic Society (ATS) and the Centers for Disease Control and Prevention was adopted by the ATS Board of Directors, July 1999. This statement was endorsed by the Council of the Infectious Diseases Society of America, September 1999. Am J Respir Crit Care Med.2000;161(4, pt 1):1376-1395.

7. Bhargava DK, Shriniwas, Chopra P, et al. Peritoneal tuberculosis: laparoscopic patterns and its diagnostic accuracy. Am J Gastroenterol. 1992:87:109-112.

8. Tang LC, Cho HK, Wong Taam VC. Atypical presentation of female genital tract tuberculosis. Eur J Obstet Gynecol Reprod Biol.1984;17:355-363.

9. K. M. Chow, V. C.-Y. Chow, and C. C. Szeto, "Indication for peritoneal biopsy in tuberculous peritonitis," American Journal of Surgery, vol. 185, no. 6, pp. 567-573, 2003.

10. A. A. Lazarus and B. Thilagar, "Abdominal Tuberculosis," Disease-aMonth, vol. 53, no. 1, pp. 32-38, 2007 